\title{
Ensuring Fairness under Prior Probability Shifts
}

\author{
Arpita Biswas $^{1}$, Suvam Mukherjee ${ }^{2}$ \\ ${ }^{1}$ Indian Institute of Science \\ ${ }^{2}$ Microsoft Research \\ arpitab@iisc.ac.in, suvamm@outlook.com
}

\begin{abstract}
In this paper, we study the problem of fair classification in the presence of prior probability shifts, where the training set distribution differs from the test set. This phenomenon can be observed in the yearly records of several real-world datasets, such as recidivism records and medical expenditure surveys. If unaccounted for, such shifts can cause the predictions of a classifier to become unfair towards specific population subgroups. While the fairness notion called Proportional Equality (PE) accounts for such shifts, a procedure to ensure PE-fairness was unknown.

In this work, we propose a method, called CAPE, which provides a comprehensive solution to the aforementioned problem. CAPE makes novel use of prevalence estimation (quantification) techniques, sampling and an ensemble of classifiers to ensure fair predictions under prior probability shifts. We introduce a metric, called prevalence difference (PD), which CAPE attempts to minimize in order to ensure PE-fairness. We theoretically establish that this metric exhibits several desirable properties.

We evaluate the efficacy of CAPE via a thorough empirical evaluation on synthetic datasets. We also compare the performance of CAPE with several popular fair classifiers on real-world datasets like COMPAS (criminal risk assessment) and MEPS (medical expenditure panel survey). The results indicate that CAPE ensures PE-fair predictions, while performing well on other performance metrics.
\end{abstract}

\section{Introduction}

Machine learning techniques are being increasingly applied in making important societal decisions, such as criminal risk assessment, school admission, hiring, sanctioning of loans, etc. Given the impact and sensitivity of such predictions, there is warranted concern regarding implicit discriminatory traits exhibited by such techniques. Such discrimination may be detrimental for certain population subgroups with a specific race, gender, ethnicity, etc, and may even be illegal under certain circumstances [Angwin et al., 2016]. These concerns have spurred vast research in the area of algorithmic fairness [Corbett-Davies and Goel, 2018; Dressel and Farid, 2018; Chouldechova and Roth, 2018; Friedler et al., 2019; Zhang et al., 2018b; Berk et al., 2018;
Kleinberg et al., 2018; Barocas and Selbst, 2016; Chouldechova, 2017; Romei and Ruggieri, 2014]. Most of these papers aim to establish fairness notions for a group of individuals (differentiated by their race, gender, etc.), and are classified as group fairness notions.

A possible, and less studied, cause for unfairness in predictions involve distributional changes (or drift) between the training and test datasets. Disparities can be introduced when the sub-populations evolve differently over time [Barocas et al., 2017]. There are important real-world scenarios where a type of distributional change, called prior probability shift, occurs. Informally, a prior probability shift occurs when the fraction of positively labeled instances differ between the training and the test datasets (see Section 2.1 for a formal definition). A concrete example is the COMPAS dataset [ProPublica, 2016] which contains demographic information and criminal history of defendants, and records whether they recommitted a crime within a certain period of time (positive labels are given to the re-offenders, while others have negative labels). We observe that, among the valid records screened in the year 2013, the fraction of Caucasian and African-American re-offenders were 0.327 and 0.486 , respectively. However, in 2014, these fractions were 0.636 and 0.706 , respectively. This indicates that the extent of prior probability shift differs among Caucasian and AfricanAmerican defendants, between the records of 2013 and 2014.

If such distributional changes are unaccounted for, a classifier may end up being unfair towards the population subgroups which exhibit prior probability shifts. For example, if the rate of recidivism among a particular sensitive group reduces drastically, then a classifier trained with a higher rate of recidivism can create extreme unfairness towards individuals of that sub-population. In this work, we address these concerns and propose a method to obtain fair predictions under prior probability shifts.

Related Work. A large body of work defines various group fairness notions and provides algorithms to mitigate unfairness. Among these, Proportional Equality (PE) [Biswas and Mukherjee, 2019; Hunter, 2000] appears to be the most appropriate fairness notion for addressing prior probability shifts among population subgroups (see Section 2.2 for definition). However, the existing results stop short of providing a procedure to ensure PE-fair predictions. We address this concern by proposing an end-to-end solution.

Apart from PE, there are other group fairness notions, none of which address prior probability shifts explicitly, such as Disparate Impact [Feldman et al., 2015; Zafar et al., 2017b; 
Kamiran and Calders, 2012; Calders et al., 2009], Statistical Parity [Corbett-Davies et al., 2017; Kamishima et al., 2012; Zemel et al., 2013], Equalized Odds [Hardt et al., 2016; Kleinberg et al., 2017; Woodworth et al., 2017], and Disparate Mistreatment [Zafar et al., 2017a].

Unfortunately, all these fairness constraints are often non-convex, thereby making the optimization problem (maximizing accuracy subject to fairness constraints) difficult to solve efficiently. Several papers provide convex surrogates of the non-convex constraints [Goh et al., 2016; Zafar et al., 2017b], or finds near-optimal near-feasible solutions [Cotter et al., 2019; Celis et al., 2019], or propose techniques to reduce dependence of group information on the predictions [Kamiran and Calders, 2012; Kamiran et al., 2012; Pleiss et al., 2017; Zhang et al., 2018a $]^{1}$. However, most of these solutions assume that the training and test datasets are identically and independently drawn from some common population distribution, and thus suffer in the presence of prior probability shifts (we provide empirical evidences in Section 4).

Our Contributions. To the best of our knowledge, we are the first to propose an end-to-end solution to ensure fair predictions in the presence of prior probability shifts.

1. We design a system called CAPE (Combinatorial Algorithm for Proportional Equality) in Section 3.

2. We introduce a metric called Prevalence Difference (PD), which CAPE attempts to minimize in order to ensure PEfairness. We theoretically establish that the PD metric exhibits several desirable properties (Theorems 1,2)—in particular, we show that maximizing the accuracy of any subgroup is not at odds with minimizing PD. This metric also provides insights into why the predictions of CAPE are fair (Theorem 3). We discuss these in Section 3.1 and 3.2.

3. We perform a thorough evaluation of CAPE on synthetic and real-world datasets, and compare with several other fair classifiers. In Section 4, we provide empirical evidence that CAPE provides PE-fair predictions, while performing well on other fairness metrics.

\section{Background and Notations}

In this paper, we focus on the binary classification problem, under prior probability shifts. Let $\hat{h}: \mathcal{X} \mapsto \mathcal{Y}$ be the prediction function, defined in some hypothesis space $\mathcal{H}$, where $\mathcal{X} \subset \mathbb{R}^{m}$ is the $m$-dimensional feature space and $\mathcal{Y}=\{0,1\}$ is the label space. The goal of a classification problem is to learn the function $\hat{h}$ which minimizes a target loss function, say, misclassification error $\mathbb{P}[\hat{h}(X) \neq Y]$ (variables $X$ and $Y$ denote feature vectors and labels). However, if these predictions $\hat{h}(\cdot)$ are used for societal decision making, it becomes crucial to ensure lower misclassification error not only on an average but also within each group defined by their sensitive attribute values such as race, gender, ethnicity, etc. Dropping these sensitive attributes blindly

\footnotetext{
${ }^{1}$ Note that the group fairness notions require the test set to be of (statistically) significant size for fairness evaluation.
}

from the dataset may not be enough to alleviate discrimination since some non-sensitive features can be closely correlated to the sensitive attributes [Zliobaite and Custers, 2016; Corbett-Davies and Goel, 2018; Hardt et al., 2016]. Hence, most existing solutions assume access to the sensitive attributes. In the presence of such a sensitive attribute with $G$ sub-populations, the goal is to learn $\hat{h}: \mathcal{X} \times[G] \mapsto \mathcal{Y}$ satisfying certain group-fairness criteria (where $[G]$ denotes the set $\{0,1, \ldots, G-1\}$ ). We use variable $Z \in[G]$ to denote group membership (one can encode multiple sensitive attributes into $[G]$ ). We assume that the training dataset $D=\left\{\left(x_{i}, z_{i}, y_{i}\right)_{i=1}^{N}\right\}$ is drawn from an unknown joint distribution $\mathcal{P}$ over $\mathcal{X} \times[G] \times \mathcal{Y}$. The performance of the classifier is measured using a new set of data, referred as test dataset $\mathbb{D}=\left\{\left(x_{j}, z_{j}, y_{j}\right)_{j=1}^{n}\right\}$, by observing how accurate and fair the $\hat{h}\left(x_{j}\right)$ s are with respect to the true labels $y_{j} \mathrm{~s}$.

Next, we focus on an important phenomenon called prior probability shift, which may cause a learned classifier to be unfair in its predictions on a test dataset.

\subsection{Prior Probability Shift}

Prior probability shift [Saerens et al., 2002; Moreno-Torres et al., 2012; Kull and Flach, 2014] occurs when the prior classprobability $\mathcal{P}(Y)$ changes between the training and test sets, but the class conditional probability $\mathcal{P}(X \mid Y)$ remains unaltered. Such changes, within a sub-population, occur in many real-world scenarios, that is, $\mathcal{P}(X \mid Y=1, Z=z)$ remains constant but $\mathcal{P}(Y=1 \mid Z=z)$ changes between the training and test datasets. If left unaccounted for, it may lead to unfair predictions [Barocas et al., 2017].

\subsection{Proportional Equality}

To address the fairness concern under prior probability shifts, a notion called proportional equality (PE) was formalized in [Biswas and Mukherjee, 2019]. A classifier is said to be PEfair if it has low values for the following expression:

$$
\mathrm{PE}^{z, z^{\prime}}:=\left|\frac{\rho_{\mathbb{D}}^{z}}{\rho_{\mathbb{D}}^{z^{\prime}}}-\frac{\hat{\rho}_{\mathbb{D}}^{z}}{\hat{\rho}_{\mathbb{D}}^{z^{\prime}}}\right| \text { for all } z, z^{\prime} \in[G]
$$

- True prevalence $\rho_{\mathbb{D}}^{z}$ is the fraction of population, from the group $z$, labeled positive in the dataset $\mathbb{D}$.

$$
\rho_{\mathbb{D}}^{z}:=\frac{\left|\left\{\left(x_{i}, z_{i}, y_{i}\right) \in \mathbb{D} \mid y_{i}=1, z_{i}=z\right\}\right|}{\left|\left\{\left(x_{i}, z_{i}, y_{i}\right) \in \mathbb{D} \mid z_{i}=z\right\}\right|} .
$$

- Prediction prevalence $\hat{\rho}_{\mathbb{D}}^{z}$ is the fraction of population, from the group $z$, predicted positive by the classifier for $\mathbb{D}$.

$$
\hat{\rho}_{\mathbb{D}}^{z}:=\frac{\left|\left\{\left(x_{i}, z_{i}, y_{i}\right) \in \mathbb{D} \mid \hat{y}_{i}=1, z_{i}=z\right\}\right|}{\left|\left\{\left(x_{i}, z_{i}, y_{i}\right) \in \mathbb{D} \mid z_{i}=z\right\}\right|} .
$$

However, Biswas and Mukherjee [2019] do not provide any algorithm for ensuring PE-fair predictions. Any such algorithm must deal with the following key challenges:

1. $\mathrm{PE}^{z, z^{\prime}} \leq \epsilon$ (for a small $\epsilon$ ) is a non-convex constraint. Thus, it is hard to directly optimize for accuracy subject to this constraint for all $z, z^{\prime} \in[G]$. 
2. The definition of PE uses true prevalences of the test datasets $\rho_{\mathbb{D}}^{z}$, which are unavailable to the classifier during the prediction phase. Thus, an algorithm needs to estimate these prevalences. Techniques from the quantification literature can be leveraged to solve this concern, which we describe next.

\subsection{Quantification Problem}

Quantification learning (or prevalence estimation) is a supervised learning problem, introduced by Forman [2005]. It aims to predict an aggregated quantity for a set of instances. The goal is to learn a function, called quantifier $q: \mathcal{X}^{\mathbb{N}} \mapsto[0,1]$, that outputs an estimate of the true prevalence of a finite, non-empty and unlabeled test set $\mathbb{D} \sim \mathcal{X}^{\mathbb{N}}$. As highlighted by Forman, quantification is not a by-product of classification [González et al., 2017]. In fact, unlike assumptions made in classification, quantification techniques account for changes in prior probabilities $\mathcal{P}(Y \mid Z)$ within subgroups, while assuming $\mathcal{P}(X \mid Y, Z)$ remain the same over the training and test datasets. This allows quantifiers to perform better than naïve classify and count techniques, as demonstrated by Forman [2006].

Some commonly used algorithms to construct quantifiers are Adjusted Classify and Count (ACC) [Forman, 2006], Scaled Probability Average (SPA) [Bella et al., 2010], and HDy [GonzáLez-Castro et al., 2013]. These algorithms can be used to estimate the prevalence of a group in the test set.

For ease of exposition, we describe a simple quantification technique, ACC. This method learns a binary classifier from the training set and estimates its true positive rates $(T P R)$ and false positive rates $(F P R)$ via $k$-fold cross-validation. Using this trained model, the algorithm counts the number of cases on which the classifier outputs positive on the test set. Finally, the true fraction of positives (true prevalence) is estimated via the equation $p=\frac{p^{\prime}-F P R}{T P R-F P R}$, where $p^{\prime}$ denotes the fraction of predicted positives, $p^{\prime}:=\frac{\# \text { predicted_positives }}{\text { \#test_data_points }}$. The use of $T P R=\frac{T P}{T P+F N}$ and $F P R=\frac{F P}{F P+T N}$ from the training set can be justified by the assumption that $P(X \mid Y)$ remains same in the training and test datasets. This simple algorithm turns out to provide good estimates of prevalences under prior probability shifts. However, for our experiments, we use SPA [Bella et al., 2010], which uses a probability estimator instead of a classifier, and turns out to be more robust to variations while estimating probabilities of a dataset with a few samples.

Next, we discuss CAPE, which provides a comprehensive solution to the above problems by combining quantification techniques along with training an ensemble of classifiers.

\section{CAPE}

In this section, we introduce CAPE (Combinatorial Algorithm for Proportional Equality), for ensuring PE-fair predictions. CAPE takes as input a training dataset $D$ and a vector of desired prevalences $\Theta=\left(\theta_{1}, \ldots, \theta_{k}\right) \in[0,1]^{k}$. CAPE is separately trained for each group $z \in[G]$, since we hypothesize that the relationship between the non-sensitive features $X$ and the outcome variable $Y$ may differ across groups. Thus, each group would be best served by training classifiers on datasets obtained from the corresponding group ${ }^{2}$. Such decoupled classifiers are also considered by Dwork et al. [2017], but they do not handle prior probability shifts.

The training phase outputs, for each group $z$, the following:

1. a set of $|\Theta|$ classifiers, each trained using a sampling of the training dataset obtained by the module PP-SAMPLING, which takes as input a prevalence parameter $\theta \in \Theta$ and a training set with $N_{z}$ data points. It randomly selects, with replacement, $\theta \times N_{z}$ instances with $Y=1$ and $(1-\theta) \times N_{z}$ instances with $Y=0$. Thus, it outputs a sample of size $N_{z}$. Each classifier is thus specialized in providing accurate predictions on datasets with particular prevalences.

2. a quantifier $\hat{q}^{z}(\cdot)$, generated by the Q-ALG module, which is subsequently used in the prediction phase of CAPE to estimate the true prevalence of the test dataset, $\rho_{\mathbb{D}}^{z}$. Separate quantifiers are created for each group since the extent of prior probability shifts may differ across groups.

During the prediction phase, for each group $z$, an estimate of the prevalence of the test data $\mathbb{D}^{z}$ is obtained using $\hat{q}^{z}(\cdot)$ (learned in the training phase). This estimate is then used to choose the classifier $J_{z}$ that minimizes the prevalence difference metric (Section 3.1). Finally, CAPE outputs the predictions of the classifier $J_{z}$ on the test set $\mathbb{D}^{z}$.

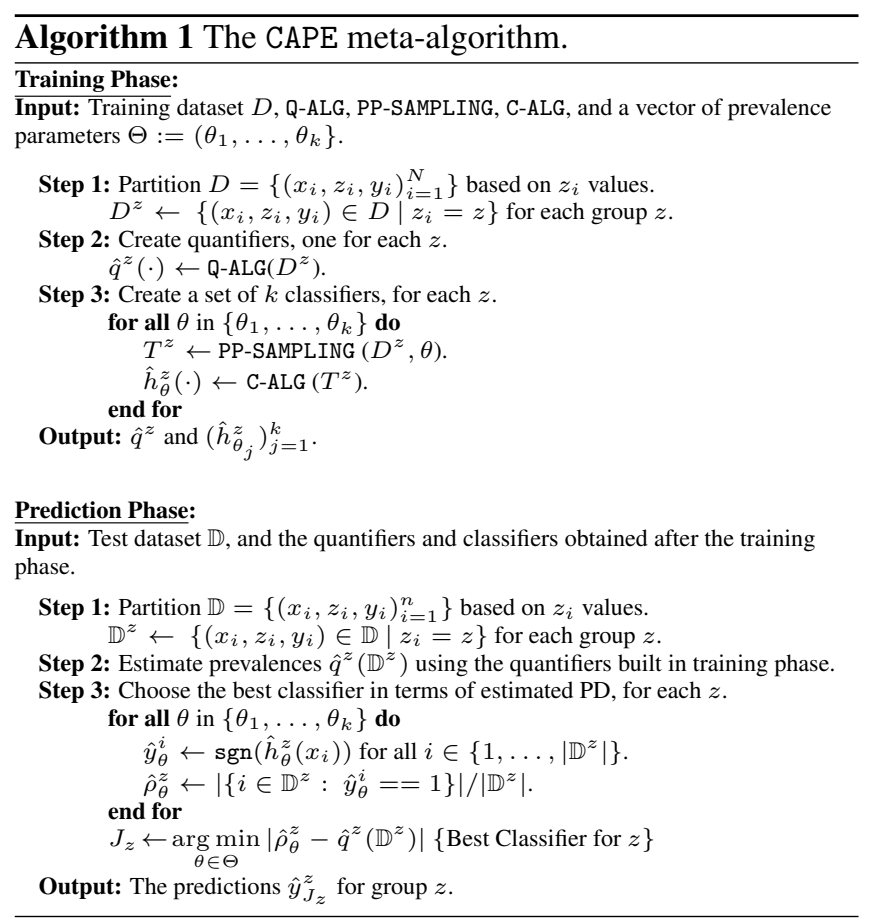

Note that CAPE provides the flexibility to plug in any classification and quantification algorithm into modules C-ALG and Q-ALG. Key to CAPE is the prevalence difference metric, used in Step 3 of the prediction phase. We formalize the metric and discuss some of its properties in the next section.

\footnotetext{
${ }^{2}$ Training a separate classifier for a small-sized subgroup may be inappropriate. For the datasets we consider, this issue never arises.
} 


\subsection{Prevalence Difference Metric}

We define the prevalence difference (PD) metric, for each group $z$, as: $\Delta_{\mathbb{D}}^{z}:=\left|\rho_{\mathbb{D}}^{z}-\hat{\rho}_{\mathbb{D}}^{z}\right|$, where, $\rho_{\mathbb{D}}^{z}$ and $\hat{\rho}_{\mathbb{D}}^{z}$ denote the true and predicted prevalences of the dataset $\mathbb{D}$ (as defined in Equations 1 and 2, respectively). Hereafter, we drop the subscripts and superscripts on $\Delta, \rho$ and $\hat{\rho}$ whenever we refer to the population in aggregate.

Note that the true prevalence $\rho_{\mathbb{D}}^{z}$ of test set $\mathbb{D}$ cannot be used during the prediction phase. Thus, replacing $\rho_{\mathbb{D}}^{z}$ with $\hat{q}^{z}\left(\mathbb{D}^{z}\right)$ in the definition of $\Delta_{\mathbb{D}}^{z}$ provides a measure to choose the best classifier $J^{z}$ for the group $z$. Also, unlike PD, other performance metrics like accuracy, FPR or FNR are not suitable for choosing the best classifier since these metrics require the true labels of the test datasets. We use the PD metric for: (1) choosing the best classifier in the prediction phase and (2) measuring the performance of the predictions, since a high value of $\Delta^{z}$ implies the inability to account for prior probability shift for the group $z$.

The PD metric is somewhat different from the fairness metrics aiming to capture parity between two sub-populations. Such fairness metrics may often require sacrificing the performance on one group to maintain parity with the other group. However PD, in itself, believes that the two groups should be treated differently since each group may have gone through a different change of prior probabilities. A high $\Delta^{z}$ indicates high extent of harm caused by the predictions made towards to the group $z$. Thus, to audit the impact of a classifier's predictions on a group $z$, it is important to evaluate for $\Delta^{z}$, along with accuracy, FNR and FPR values within each group.

Next, we show that a perfect classifier (100\% accurate) attains zero prevalence difference. Additionally, we show that a classifier with high accuracy on any sub-group also attains a very low $\Delta$ for that subgroup. Empirically, we observe that low $\Delta$ results in PE-fair predictions.

\subsection{Theoretical Guarantees}

We first show a simple result- a classifier whose predictions are exactly the ground truth also attains $\Delta=0$, thereby satisfying our proposed metric used for selecting the best classifier. Note that a perfect classifier may not satisfy fairness notions such as disparate impact and statistical parity.

Theorem 1. A perfect classifier always exhibits $\Delta=0$.

Proof. Let us consider a perfect classifier $C$ whose predictions are equal to the ground truth i.e., $\hat{y}(x)=y(x)$ for all instances $x \in \mathcal{X}$, where $\hat{y}(x)$ is the label predicted by the classifier $C$ for the instance $x$. Thus, for each $z$, the true prevalence $\rho^{z}$ is equal to the prediction prevalence $\hat{\rho}^{z}$, according to the definitions in Equations 1 and 2. Thus, the prevalence difference $\Delta^{z}=\left|\rho^{z}-\hat{\rho}^{z}\right|=0$.

Theorem 2. If the overall accuracy of a classifier $C$ is $(1-\delta)$, where $\delta \in(0,1)$ is a very small number, then the overall prevalence difference for $C$ is $\Delta=\delta-2 \min \left\{\frac{\mathrm{FN}}{n}, \frac{\mathrm{FP}}{n}\right\}$, where FN and FP denote number of false negatives and false positives respectively in the test dataset with $n$ instances. This further implies that $\Delta \leq \delta$.
Proof. Let $\left(\hat{y}_{i}\right)_{i=1}^{n}$ denote the predictions of a classifier $C$ on a test dataset $\left\{\left(x_{i}, y_{i}\right)_{i=1}^{n}\right\}$. Some other notations that we use for the proof, are:

$\mathrm{TP}:=\left|\left\{i: y_{i}=1 \& \hat{y}_{i}=1\right\}\right|$ (\# true positives).

$\mathrm{TN}:=\mid\left\{i:\left(y_{i}=0 \& \hat{y}_{i}=0\right\} \mid\right.$ (\# true negatives).

$\mathrm{FP}:=\left|\left\{i: y_{i}=0 \& \hat{y}_{i}=1\right\}\right|$ (\# false positives).

FN $:=\left|\left\{i: y_{i}=1 \& \hat{y}_{i}=0\right\}\right|$ (\# false negatives).

Note that $\mathrm{TP}+\mathrm{TN}+\mathrm{FP}+\mathrm{FN}=n$. Let $\rho$ and $\hat{\rho}$ be the true and prediction prevalences. Then, the prevalence difference can be written as:

$$
\Delta=|\rho-\hat{\rho}|=\left|\frac{\mathrm{TP}+\mathrm{FN}}{n}-\frac{\mathrm{TP}+\mathrm{FP}}{n}\right|=\frac{|\mathrm{FN}-\mathrm{FP}|}{n}
$$

Let the accuracy of a classifier on a test dataset be $(1-\delta)$ where $\delta \in(0,1)$. Then,

$$
\frac{\mathrm{TP}+\mathrm{TN}}{n}=1-\delta \Rightarrow \frac{\mathrm{FN}+\mathrm{FP}}{n}=\delta
$$

Without loss of generality, let us assume FN $\geq$ FP. Thus, Equation 4 can be written as:

$$
\frac{\mathrm{FN}-\mathrm{FP}+2 \mathrm{FP}}{n}=\delta \Rightarrow \frac{\mathrm{FN}-\mathrm{FP}}{n}=\delta-\frac{2 \mathrm{FP}}{n}
$$

Similarly, assuming FP $\geq$ FN we obtain

$$
\frac{\mathrm{FP}-\mathrm{FN}}{n}=\delta-\frac{2 \mathrm{FN}}{n}
$$

Combining Equation 3, 5 and 6, we get the following:

$$
\begin{aligned}
\Delta & =\frac{|\mathrm{FP}-\mathrm{FN}|}{n}=\delta-2 \min \left\{\frac{\mathrm{FN}}{n}, \frac{\mathrm{FP}}{n}\right\} \\
\Rightarrow \Delta & \leq \delta .
\end{aligned}
$$

Thus, when accuracy is greater than $(1-\delta)$, the prevalence difference is at most $\delta$. This completes the proof.

Note that Theorem 2 can also be used to guarantee that highly accurate predictions for a group $z$, implies a low value for $\Delta^{z}$. This leads to Corollary 2.1.

Corollary 2.1. If accuracy of a classifier for any subpopulation $z$ is greater than $1-\delta$, then $\Delta^{z} \leq \delta$.

The following theorem gives insight on why CAPE works. In the subsequent discussion, we drop the parameter $\mathbb{D}$ from the notations $\hat{q}$ and $\rho$ and $\hat{\rho}$ since we exclusively refer to these values in the context of the test dataset $\mathbb{D}$ only.

Theorem 3. Let $\Theta=\left\{\frac{\epsilon}{2}, \frac{3 \epsilon}{2}, \frac{5 \epsilon}{2} \ldots,\left(k-\frac{1}{2}\right) \epsilon\right\}$ where $\epsilon \epsilon$ $(0,1)$ and $k=\left\lfloor\frac{1}{\epsilon}+\frac{1}{2}\right\rfloor$. For a group $z$, and test dataset $\mathbb{D}$, let the quantifier be such that $\left|\rho^{z}-\hat{q}^{z}\right| \leq \delta_{1}$, and the classifiers be such that $\left|\theta_{j}-\hat{\rho}_{j}^{z}\right| \leq \delta_{2}$ for all $j \in\{1, \ldots, k\}$, for small $\delta_{1}$ and $\delta_{2}$. Then, for the best classifier

$$
J:=\underset{j \in\{1, \ldots, k\}}{\arg \min }\left|\hat{\rho}_{j}^{z}-\hat{q}^{z}\right|,
$$

the following holds:

$$
\left|\rho^{z}-\hat{\rho}_{J}^{z}\right| \leq \delta_{1}+\delta_{2}+\frac{\epsilon}{2}
$$


Proof. For the best classifier $J$, the prevalence difference of a group $z$ can be upper bounded using triangle inequality:

$$
\begin{aligned}
\left|\rho^{z}-\hat{\rho}_{J}^{z}\right| & \leq\left|\rho^{z}-\hat{q}^{z}\right|+\left|\hat{q}^{z}-\hat{\rho}_{J}^{z}\right| \\
& \leq \delta_{1}+\left|\hat{q}^{z}-\hat{\rho}_{J}^{z}\right|
\end{aligned}
$$

Inequality (8) is implied by the assumption on the quantifier's performance, i.e., $\left|\rho^{z}-\hat{q}^{z}\right| \leq \delta_{1}$. To provide an upper bound for $\left|\hat{q}^{z}-\hat{\rho}_{J}^{z}\right|$, we pick $J^{\prime}$ such that

$$
J^{\prime}=\underset{j \in\{1, \ldots, k\}}{\arg \min }\left|\hat{q}^{z}-\theta_{J^{\prime}}\right|, \quad \text { where } \theta_{J^{\prime}}=\left(J^{\prime}-\frac{1}{2}\right) \epsilon
$$

Since $\hat{q}^{z} \in[0,1]$, it is at most $\epsilon / 2$ away from one of the fractional values in $\left\{\frac{\epsilon}{2}, \frac{3 \epsilon}{2}, \frac{5 \epsilon}{2} \ldots,\left(k-\frac{1}{2}\right) \epsilon\right\}$. Therefore,

$$
\left|\hat{q}^{z}-\theta_{J^{\prime}}\right| \leq \epsilon / 2
$$

We use Inequality (9) to provide an upper bound to the expression $\left|\hat{q}^{z}-\hat{\rho}_{J}^{z}\right|$, using case-by-case analysis.

Case 1: Assume $\hat{q}^{z}<\hat{\rho}_{J}^{z}$. This leaves us with three possibilities for the value of $\theta_{J^{\prime}}$ :

1. Assume $\theta_{J^{\prime}} \geq \hat{\rho}_{J}^{z}$. Then,

$$
\hat{\rho}_{J}^{z}-\hat{q}^{z} \leq \theta_{J^{\prime}}-\hat{q}^{z} \leq \epsilon / 2
$$

2. Assume $\hat{q}^{z} \leq \theta_{J^{\prime}}<\hat{\rho}_{J}^{z}$. Now, we bound the desired quantity using the value of $\hat{\rho}_{J^{\prime}}$. Note that $\left|\hat{q}^{z}-\hat{\rho}_{J}\right| \leq$ $\left|\hat{q}^{z}-\hat{\rho}_{J^{\prime}}\right|$ since $J$ is the best classifier. Thus, either $\hat{\rho}_{J^{\prime}} \geq$ $\hat{\rho}_{J}$ or $\hat{\rho}_{J^{\prime}} \leq \hat{q}^{z}$.

(a) Assume $\hat{\rho}_{J^{\prime}} \leq \hat{q}^{z}$. Then,

$$
\left|\hat{q}^{z}-\hat{\rho}_{J}^{z}\right| \leq \hat{q}^{z}-\hat{\rho}_{J^{\prime}}^{z} \leq \theta_{J^{\prime}}-\hat{\rho}_{J^{\prime}}^{z} \leq \delta_{2}
$$

(b) Assume $\hat{\rho}_{J^{\prime}} \geq \hat{\rho}_{J}$. Then,

$$
\begin{aligned}
\left|\hat{q}^{z}-\hat{\rho}_{J}^{z}\right| & \leq\left(\hat{\rho}_{J^{\prime}}^{z}-\hat{q}^{z}\right) \\
& =\left(\hat{\rho}_{J^{\prime}}^{z}-\theta_{J^{\prime}}\right)+\left(\theta_{J^{\prime}}-\hat{q}^{z}\right) \\
& \leq \delta_{2}+\epsilon / 2
\end{aligned}
$$

3. Assume $\theta_{J^{\prime}}<\hat{q}^{z}$. Now, we bound the desired quantity using the value of $\hat{\rho}_{J^{\prime}}$, and there can be three cases.

(a) Assume $\hat{\rho}_{J^{\prime}} \leq \theta_{J^{\prime}}$. Then,

$$
\begin{aligned}
\left|\hat{q}^{z}-\hat{\rho}_{J}^{z}\right| & \leq \hat{q}^{z}-\hat{\rho}_{J^{\prime}}^{z} \\
& \leq\left(\hat{q}^{z}-\theta_{J^{\prime}}\right)+\left(\theta_{J^{\prime}}-\hat{\rho}_{J^{\prime}}^{z}\right) \\
& \leq \epsilon / 2+\delta_{2}
\end{aligned}
$$

(b) Assume $\theta_{J^{\prime}}<\hat{\rho}_{J^{\prime}} \leq \hat{q}^{z}$. Then,

$$
\begin{aligned}
\left|\hat{q}^{z}-\hat{\rho}_{J}^{z}\right| \leq \hat{q}^{z}-\hat{\rho}_{J^{\prime}}^{z} & \leq \hat{q}^{z}-\theta_{J^{\prime}} \\
& \leq \epsilon / 2
\end{aligned}
$$

(c) Assume $\hat{\rho}_{J^{\prime}}>\hat{\rho}_{J}$. Then,

$$
\left|\hat{q}^{z}-\hat{\rho}_{J}^{z}\right| \leq \hat{\rho}_{J^{\prime}}^{z}-\theta_{J^{\prime}} \leq \delta_{2}
$$

Inequalities (10)-(15) establish the following upper bound when $\hat{q}^{z}<\hat{\rho}_{J}^{z}$,

$$
\left|\hat{q}^{z}-\hat{\rho}_{J}^{z}\right| \leq \delta_{2}+\epsilon / 2 .
$$

Case 2: $\hat{q}^{z} \geq \hat{\rho}_{J}^{z}$. An analysis analogous to Case 1 gives the same inequality as (16). Combining Inequalities (8) and (16), we obtain the desired upper bound of $\delta_{1}+\delta_{2}+\epsilon / 2$ on the quantity $\left|\rho^{z}-\hat{\rho}^{z}\right|$.

\section{Experimental Evaluation}

We first evaluate CAPE on synthetically generated datasets. We then compare it with other fair classifiers on the realworld COMPAS [ProPublica, 2016] and MEPS [Agency for Healthcare Research \& Quality, 2016] datasets, where we observe possible prior-probability shifts. CAPE is open source but the link is retracted for anonymity. The performance of CAPE on a wide range of fairness-metrics, across all these datasets, enforces our proposal that CAPE should be used for predictions under prior-probability shifts.

\subsection{Datasets}

Synthetic: We assume a generative model with 3 featuressensitive attribute $Z \in\{0,1\}$, and two additional attributes $U$ and $V$-along with the label $Y \in\{0,1\}$. We assume that the overall population distribution is generated as $\mathcal{P}(U, V, Z, Y)=\mathcal{P}(U, V \mid Z, Y) \cdot \mathcal{P}(Z \mid Y) \cdot \mathcal{P}(Y)$. We further consider equal representation of the two population subgroups, i.e., $\mathcal{P}(Z=1 \mid Y)=\mathcal{P}(Z=0 \mid Y)$ for each $Y \in\{0,1\}$. $U$ and $V$ are conditionally independent: $\mathcal{P}(U, V \mid Z, Y)=$ $\mathcal{P}(U, V \mid Y)=\mathcal{P}(U \mid Y) \cdot \mathcal{P}(V \mid Y)$, and the distributions are considered to be Gaussian $(\mathcal{N})$ with the following parameters: $\mathcal{P}(U \mid Y=1) \sim \mathcal{N}(15,10), \mathcal{P}(U \mid Y=0) \sim \mathcal{N}(5,5)$, $\mathcal{P}(V \mid Y=1) \sim \mathcal{N}(20,10)$, and $\mathcal{P}(V \mid Y=0) \sim \mathcal{N}(40,10)$.

We generate 50000 instances for the training dataset $D$ with equal label distribution, i.e., $\rho_{D}^{z}=0.5$. However, while generating the test set, the prevalence parameters $\rho_{\mathbb{D}}^{z}$ are different. We generated 81 different types of test datasets, each obtained by varying the prevalences for both subgroups $z \in\{0,1\}$, such that $\rho_{\mathbb{D}}^{z} \in\{0.1, \ldots, 0.9\}$.

COMPAS dataset contains demographic information and criminal history for pre-trial defendants in Broward County, Florida. The goal of learning is to predict whether an individual re-offends. We consider is_recid as $Y$ labels and race as the sensitive attribute $(Z=1$ denotes African-Americans, while $Z=0$ denotes Caucasians). We pre-processed the dataset to remove rows containing missing or invalid information. Our training dataset comprises 4278 records whose screening dates were in the year 2013 (of which $59.70 \%$ are African-Americans), while the test dataset comprises 1809 records screened in the year 2014 (of which $60.86 \%$ are African-Americans).

MEPS comprises surveys carried out on individuals, health care professions, and employers in the United States. The feature UTILIZATION measures the total number of trips involved in availing some sort of medical facility. The classification task involves predicting whether UTILIZATION $\geq 10$. We consider RACE as the sensitive attribute $(Z=1$ denotes 'Non-Whites'). The surveys for the year 2015 is our training set (with 33400 data points, of which $62.86 \%$ are 'NonWhites'), and the surveys for 2016 is our test set (with 32006 data points, of which $61.72 \%$ are 'Non-Whites').

\subsection{Other Algorithms for Comparison}

We compare CAPE against an accuracy-maximizing classifier, Max_Acc. It uses the same algorithm used by CAPE 
in the module C-ALG. On the real-world datasets, we additionally compare CAPE with the following (in-, preand post-processing) fair algorithms, implemented in the IBM AI Fairness 360 [Bellamy et al., 2018] toolkitReweighing (Reweigh) [Kamiran and Calders, 2012], Adversarial Debiasing (AD) [Zhang et al., 2018a], and variants of Meta_fair [Celis et al., 2019], Calibrated Equalized Odds Postprocessing (CEOP) [Pleiss et al., 2017], Reject Option Classification (ROC) [Kamiran et al., 2012]. These algorithms target fairness notions other than PE. We evaluate the extent to which these algorithms achieve PE fairness and compare how they perform on a set of other metrics (such as FPR-diff, FNR-diff, Accuracy-diff, and PD). While CAPE can handle multiple sensitive attributes, we choose one sensitive attribute for all the datasets to stay consistent with the implementation in the IBM AIF360 toolkit.

\subsection{Parameters and Modules used for CAPE}

- Prevalences: We set $\Theta=\{0.05,0.15, \ldots, 0.95\}$.

- PP-SAMPLING: As described in Section 3.

- Q-ALG: Scaled Probability Average [Bella et al., 2010].

- C-ALG: As the synthetically generated datasets are created using simple generative models, we use generalized logistic regression (glm) with regularization. For COMPAS and MEPS, we use gradient boosted algorithm (gbm) and 10fold cross-validation for hyper-parameter tuning.

\subsection{Results}

Synthetic dataset: We evaluated CAPE with 81 types of test datasets, each with $\rho_{\mathbb{D}}^{z} \in\{0.1, \ldots, 0.9\}$ for $z \in\{0,1\}$. The general trend we observe is that CAPE outperforms Max_Acc whenever there is a significant shift in prior probabilities. We report two interesting sets of results here.

First, we consider test datasets with $\rho_{\mathbb{D}}^{0}=0.5$, and $\rho_{\mathbb{D}}^{1}$ ranging between 0.1 and 0.9 . Figure 1 summarizes our findings. Since CAPE accounts for prevalence changes, the accuracy of CAPE on $\mathbb{D}$ for group $Z=1$ (Figure 1a) is consistently higher than Max_Acc, except for the dataset with $\rho_{\mathbb{D}}^{1}=0.5$ where the accuracies become nearly equal. The prevalence difference for $Z=1$ (Figure 1b) is lower for CAPE whenever there is a prior probability shift (i.e., when $\rho_{\mathbb{D}}^{1} \neq 0.5$ ). In fact, for Max_Acc, $\hat{\rho}_{\mathbb{D}}^{1}$ remains 0.5 across all the test datasets. Thus, $\Delta_{\mathbb{D}}^{1}$ for Max_Acc increases linearly as $\rho_{\mathbb{D}}^{1}$ moves away from
0.5. Lastly, the predictions of CAPE consistently exhibit a lower valuation for PE (Figure 1c), compared to Max_Acc. This highlights that the predictions of CAPE are more fair, compared to the purely accuracy maximizing Max_Acc.

Second, in Table 1, we report results for scenarios where both $\rho_{\mathbb{D}}^{0}$ and $\rho_{\mathbb{D}}^{1}$ significantly deviate from their corresponding prevalences in the training set. The results are representative of the general trend we observed in the other test datasetsCAPE outperforms Max_Acc on accuracy, PD and PE metrics.

\begin{tabular}{|c|c|c|c|c|c|c|c|}
\hline \multirow[b]{2}{*}{$\mathbf{Z}$} & \multirow[b]{2}{*}{$\rho_{\mathbb{D}}^{z}$} & \multicolumn{2}{|c|}{ Accuracy } & \multicolumn{2}{|c|}{$\Delta$} & \multicolumn{2}{|c|}{$\mathbf{P E} \mathbf{E}^{0,1}$} \\
\hline & & CAPE & Max_Acc & CAPE & Max_Acc & CAPE & Max_Acc \\
\hline 0 & 0.1 & 0.940 & 0.880 & 0.009 & 0.094 & \multirow{2}{*}{0.050} & \multirow{2}{*}{0.104} \\
\hline 1 & 0.1 & 0.930 & 0.855 & 0.016 & 0.110 & & \\
\hline 0 & 0.2 & 0.894 & 0.855 & 0.017 & 0.084 & \multirow{2}{*}{0.012} & \multirow{2}{*}{0.140} \\
\hline 1 & 0.8 & 0.909 & 0.877 & 0.006 & 0.074 & & \\
\hline 0 & 0.9 & 0.929 & 0.851 & 0.012 & 0.120 & \multirow{2}{*}{0.003} & \multirow{2}{*}{0.028} \\
\hline 1 & 0.9 & 0.940 & 0.879 & 0.006 & 0.097 & & \\
\hline
\end{tabular}

Table 1: Accuracy, $\Delta$ and PE values on the synthetic datasets when test set $\mathbb{D}$ is such that $\rho_{\mathbb{D}}^{z} \neq 0.5$, for both groups $z \in\{0,1\}$.

Real-world datasets: For COMPAS, columns 3 and 4 of Table 2 highlight that the true prevalences of the training (year 2013) and test (year 2014) datasets are significantly different. This is indicative of a possible prior probability shift. Column 5 shows that the Q-ALG module of CAPE makes a good estimate of the true prevalences of the test dataset.

\begin{tabular}{ccccc} 
& $\mathbf{Z}$ & $\begin{array}{c}\text { Training Data } \\
\text { True Prevalence } \\
\rho_{D}^{z}\end{array}$ & $\begin{array}{c}\text { Test Data } \\
\text { True Prevalence } \\
\rho_{\mathbb{D}}^{z}\end{array}$ & $\begin{array}{c}\text { Quantifier's } \\
\text { Estimate } \\
\left.\hat{q}^{(} \mathbb{D}^{z}\right)\end{array}$ \\
\hline \multirow{2}{*}{ COMPAS } & $\mathbf{0}$ & 0.327 & 0.636 & 0.592 \\
& $\mathbf{1}$ & 0.486 & 0.706 & 0.644 \\
\hline \multirow{2}{*}{ MEPS } & $\mathbf{0}$ & 0.253 & 0.253 & 0.273 \\
& $\mathbf{1}$ & 0.124 & 0.117 & 0.123 \\
\hline
\end{tabular}

Table 2: Column 3 and 4 show possible prior probability shifts in COMPAS and MEPS. Column 5 highlights the prevalence estimates obtained by Q-ALG module of CAPE on the test datasets.

For MEPS, we observe a shift only for the group $Z=1$, between the training set (surveys in the year 2015) and test set (surveys in 2016). Since the differences in prevalences are rather small, this dataset is of interest-it allows us to in-

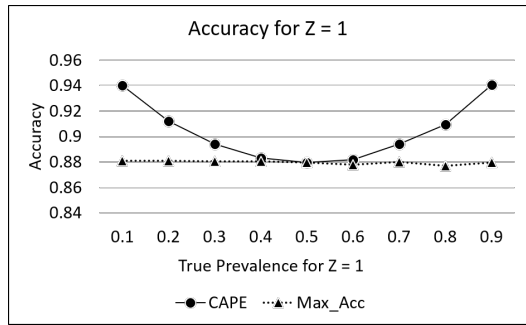

(a) Accuracy of $Z=1$.

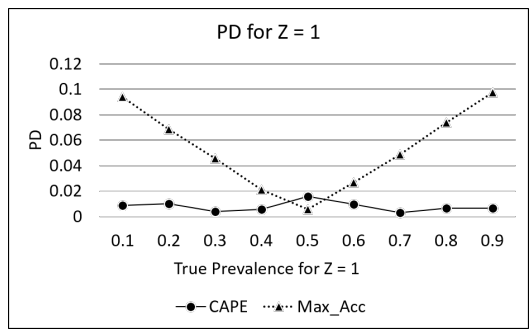

(b) Prevalence Difference for $Z=1$.

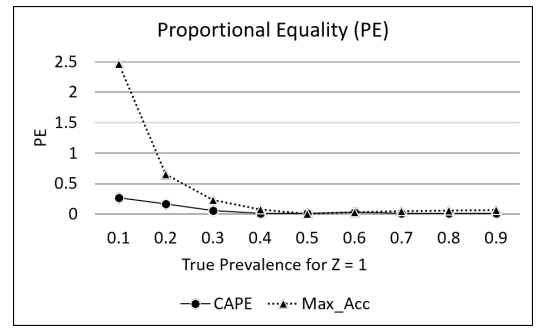

(c) Proportional Equality $\left(\mathrm{PE}^{0,1}\right)$.

Figure 1: Comparing accuracy, $\mathrm{PD}$ and PE metrics on synthetic test datasets with varying prevalences for group $Z=1$. The prevalence for group $Z=0$ is fixed at 0.5 . The reported results are averaged over 20 iterations and the standard deviation is of the order $10^{-3}$. 


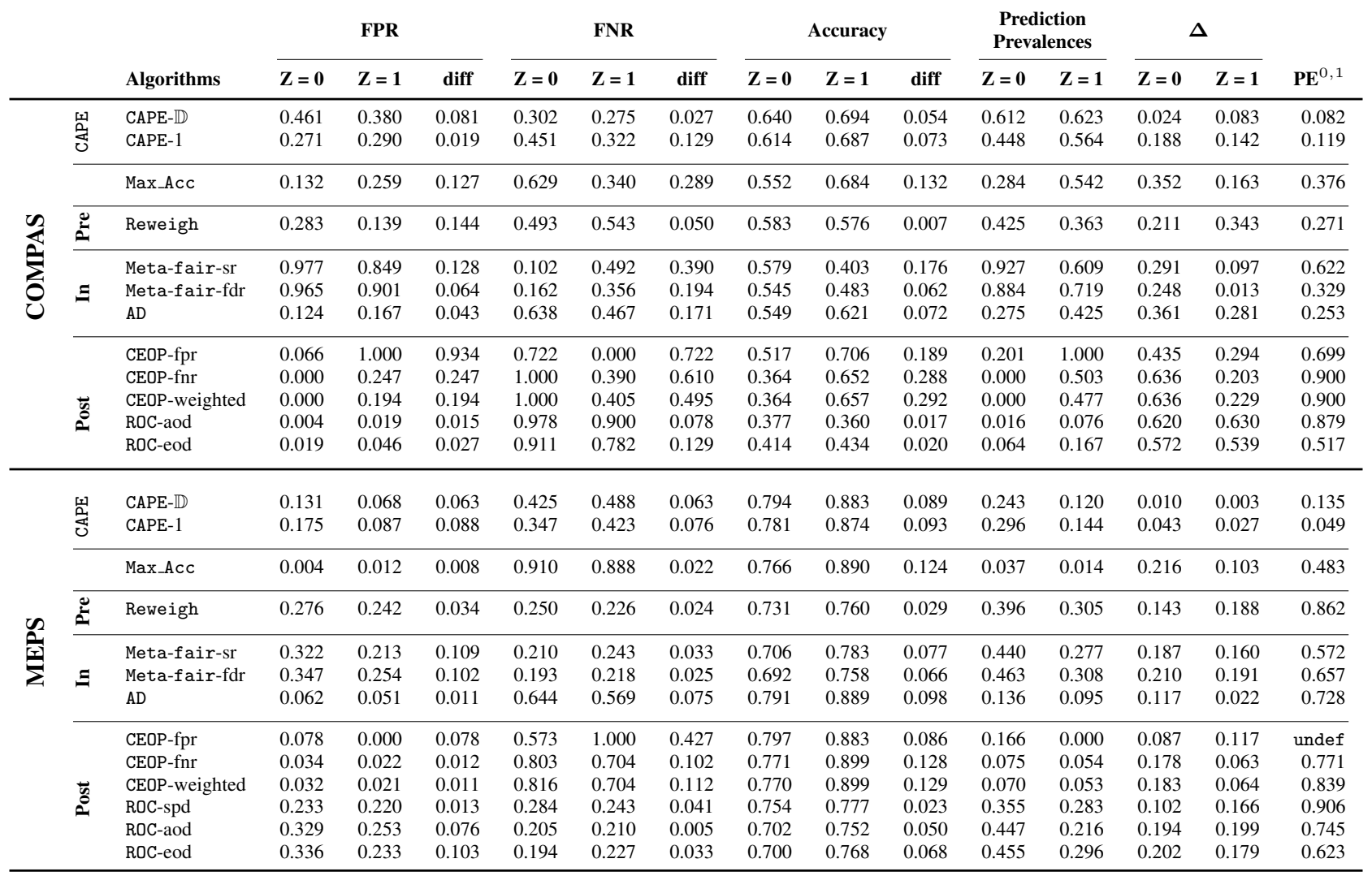

Table 3: Comparing CAPE with Max_Acc and other fair classifiers on the COMPAS and MEPS test datasets.

vestigate the performance of CAPE when the extent of prior probability shift is small. Though the prevalences estimated by Q-ALG seem similar to the training set, the difference in the estimates of Q-ALG and the prevalences of the test datasets are only 0.02 and 0.006 , for $Z=0$ and $Z=1$ respectively, and are thus good estimates.

Table 3 summarizes the results on COMPAS and MEPS datasets for CAPE, Max_Acc, and the other fair algorithms described in Section 4.2. Due to lack of space, we elaborate upon the results of the COMPAS dataset only.

CAPE- $\mathbb{D}$ considers the whole test dataset $\mathbb{D}$ during prediction, while CAPE-1 considers individual instances during prediction (similar to what the other algorithms do). We expect CAPE- $\mathbb{D}$ to perform better than CAPE-1 since the Q-ALG module is expected to perform better for larger test datasets.

CAPE-D outperforms Max_Acc on $\Delta$, and all the other fairness metrics (FPR-diff, FNR-diff, Accuracy-diff, and PE). The prediction prevalences of Max_Acc $(0.284$ and 0.542$)$ are close to the true prevalences of the training set $(0.327$ and 0.486), which highlights the inability of Max_Acc to account for the prior probability shift. One critical observation about CAPE-D is that FPR-diff $=0.081$ and FNR-diff $=0.027$ which implies that the predictions exhibit equalized odds. In comparison, these differences for Max_Acc are 0.127 and 0.289. In fact, for Max_Acc, $\mathrm{FPR}_{Z=1}$ is almost twice than $\mathrm{FPR}_{Z=0}$, whereas $\mathrm{FNR}_{Z=1}$ is almost half of $\mathrm{FNR}_{Z=0}$. This implies that Max_Acc imposes unfair higher risks of recidivism on
African-American defendants, while Caucasian defendants are predicted to have lower risks than they actually do.

The true prevalences of the two subgroups in the test dataset are close to each other (namely, 0.636 and 0.706). Thus, a classifier aiming to achieve statistical parity is expected to do well on PE. We observe this in Table 3, where ROC-spd (statistical parity difference) has lowest PE (0.056). However, its false positive rates are more than 0.9 for both subgroups, which is unfair and harmful for both subgroups. This unfairness is also captured by the high $\Delta$ value of ROCspd. We observe that $\Delta^{0}$ is the lowest for CAPE-D among all other classifiers. For $Z=1$, Meta_fair-fdr (false discovery rate with the group fairness trade-off parameter $\tau$ set to 0.8 ), is the only other fair classifier with a lower $\Delta^{1}$ value. However, the predictions of Meta_fair-fdr have high false positive rates, and low accuracies.

Note that a trivial classifier, which always predicts positive labels, will have FNR-diff=0, FPR-diff=0, Accuracydiff $=0.07$. However, this classifier will have high PD for both groups $\left(\Delta^{0}=0.364\right.$ and $\left.\Delta^{1}=0.294\right)$, which indicates a substantial skew between the false positives and false negatives. Thus, PD is an important metric that, in addition to accuracy, captures the learning ability of the classifiers.

We make a final observation on our experimental results. Since both COMPAS and MEPS are real-world datasets, the distributional changes highlighted in Table 2 may not be due to prior probability shifts alone. Although CAPE is designed to 
handle only prior probability shifts, the good performance of both CAPE-D and CAPE-1 on a wide range of metrics for these real-world datasets shows the robustness of our approach.

A possible extension of CAPE includes handling other distributional changes, such as concept drifts, that is, when $\mathcal{P}(X \mid Y, Z)$ changes but $\mathcal{P}(Y \mid Z)$ remains same.

\subsection{Acknowledgment}

Arpita Biswas gratefully acknowledges the support of a Google PhD Fellowship Award.

\section{References}

[Agency for Healthcare Research \& Quality, 2016] Agency for Healthcare Research \& Quality. Medical Expenditure Panel Survey. https://meps.ahrq.gov/mepsweb/, 2016.

[Angwin et al., 2016] Julia Angwin, Jeff Larson, Surya Mattu, and Lauren Kirchner. Machine bias: There's software used across the country to predict future criminals. and it's biased against blacks. ProPublica, 2016

[Barocas and Selbst, 2016] Solon Barocas and Andrew D Selbst. Big data's disparate impact. Cal. L. Rev., 104:671, 2016.

[Barocas et al., 2017] Solon Barocas, Moritz Hardt, and Arvind Narayanan. Fairness in machine learning. NIPS Tutorial, 2017.

[Bella et al., 2010] Antonio Bella, Cesar Ferri, José Hernández-Orallo, and Maria Jose Ramirez-Quintana. Quantification via probability estimators. In Data Mining (ICDM), 2010 IEEE 10th International Conference on, pages 737-742. IEEE, 2010.

[Bellamy et al., 2018] Rachel K. E. Bellamy, Kuntal Dey, Michael Hind, Samuel C. Hoffman, Stephanie Houde, Kalapriya Kannan, Pranay Lohia, Jacquelyn Martino, Sameep Mehta, Aleksandra Mojsilovic, Seema Nagar, Karthikeyan Natesan Ramamurthy, John Richards, Diptikalyan Saha, Prasanna Sattigeri, Moninder Singh, Kush R. Varshney, and Yunfeng Zhang. AI Fairness 360: An extensible toolkit for detecting, understanding, and mitigating unwanted algorithmic bias, October 2018.

[Berk et al., 2018] Richard Berk, Hoda Heidari, Shahin Jabbari, Michael Kearns, and Aaron Roth. Fairness in criminal justice risk assessments: The state of the art. Sociological Methods \& Research, 2018.

[Biswas and Mukherjee, 2019] Arpita Biswas and Suvam Mukherjee. Fairness through the lens of proportional equality. In Proceedings of the 18th International Conference on Autonomous Agents and MultiAgent Systems, AAMAS '19, pages 1832-1834, 2019.

[Calders et al., 2009] Toon Calders, Faisal Kamiran, and Mykola Pechenizkiy. Building classifiers with independency constraints. In Data mining workshops, 2009. ICDMW'09. IEEE international conference on, pages 13-18. IEEE, 2009.

[Celis et al., 2019] L. Elisa Celis, Lingxiao Huang, Vijay Keswani, and Nisheeth K. Vishnoi. Classification with fairness constraints: A meta-algorithm with provable guarantees. In Proceedings of the Conference on Fairness, Accountability, and Transparency, FAT*' 19, pages 319-328. ACM, 2019.

[Chouldechova and Roth, 2018] Alexandra Chouldechova and Aaron Roth. The frontiers of fairness in machine learning. arXiv preprint arXiv:1810.08810, 2018.

[Chouldechova, 2017] Alexandra Chouldechova. Fair prediction with disparate impact: A study of bias in recidivism prediction instruments. Big data, 5(2):153-163, 2017.

[Corbett-Davies and Goel, 2018] Sam Corbett-Davies and Sharad Goel. The measure and mismeasure of fairness: A critical review of fair machine learning. arXiv preprint arXiv:1808.00023, 2018

[Corbett-Davies et al., 2017] Sam Corbett-Davies, Emma Pierson, Avi Feller, Sharad Goel, and Aziz Huq. Algorithmic decision making and the cost of fairness. In Proceedings of the 23rd ACM SIGKDD International Conference on Knowledge Discovery and Data Mining, pages 797-806. ACM, 2017.

[Cotter et al., 2019] Andrew Cotter, Maya Gupta, Heinrich Jiang, Nathan Srebro, Karthik Sridharan, Serena Wang, Blake Woodworth, and Seungil You. Training well-generalizing classifiers for fairness metrics and other data-dependent constraints. In International Conference on Machine Learning, pages 1397-1405, 2019.

[Dressel and Farid, 2018] Julia Dressel and Hany Farid. The accuracy, fairness, and limits of predicting recidivism. Science advances, 4(1):eaao5580, 2018

[Dwork et al., 2017] Cynthia Dwork, Nicole Immorlica, Adam Tauman Kalai, and Max Leiserson. Decoupled classifiers for fair and efficient machine learning. arXiv preprint arXiv:1707.06613, 2017
[Feldman et al., 2015] Michael Feldman, Sorelle A Friedler, John Moeller, Carlos Scheidegger, and Suresh Venkatasubramanian. Certifying and removing disparate impact. In ACM SIGKDD International Conference on Knowledge Discovery and Data Mining, pages 259-268. ACM, 2015.

[Forman, 2005] George Forman. Counting positives accurately despite inaccurate classification. In European Conference on Machine Learning, pages 564-575. Springer, 2005

[Forman, 2006] George Forman. Quantifying trends accurately despite classifier error and class imbalance. In ACM SIGKDD international conference on Knowledge discovery and data mining, pages 157-166. ACM, 2006.

[Friedler et al., 2019] Sorelle A Friedler, Carlos Scheidegger, Suresh Venkatasubramanian, Sonam Choudhary, Evan P Hamilton, and Derek Roth. A comparative study of fairness-enhancing interventions in machine learning. In Proceedings of the Conference on Fairness, Accountability, and Transparency, pages 329-338. ACM, 2019.

[Goh et al., 2016] Gabriel Goh, Andrew Cotter, Maya Gupta, and Michael P Friedlander. Satisfying real-world goals with dataset constraints. In Advances in Neural Information Processing Systems, pages 2415-2423, 2016.

[González et al., 2017] Pablo González, Jorge Díez, Nitesh Chawla, and Juan José de Coz. Why is quantification an interesting learning problem? Progress in Artificial Intelligence, 6(1):53-58, 2017.

[GonzáLez-Castro et al., 2013] VíCtor GonzáLez-Castro, RocíO Alaiz-RodríGuez, and Enrique Alegre. Class distribution estimation based on the hellinger distance. Information Sciences, 218:146-164, 2013.

[Hardt et al., 2016] Moritz Hardt, Eric Price, Nati Srebro, et al. Equality of opportunity in supervised learning. In Advances in neural information processing systems, pages 3315-3323, 2016

[Hunter, 2000] Nan D Hunter. Proportional equality: Readings of romer. Ky. $L J$, $89: 885,2000$

[Kamiran and Calders, 2012] Faisal Kamiran and Toon Calders. Data preprocessing techniques for classification without discrimination. Knowledge and Information Systems, 33(1):1-33, 2012.

[Kamiran et al., 2012] Faisal Kamiran, Asim Karim, and Xiangliang Zhang. Decision theory for discrimination-aware classification. In ICDM, pages 924-929. IEEE Computer Society, 2012.

[Kamishima et al., 2012] Toshihiro Kamishima, Shotaro Akaho, Hideki Asoh, and Jun Sakuma. Fairness-aware classifier with prejudice remover regularizer. In Joint European Conference on Machine Learning and Knowledge Discovery in Databases, pages 35-50. Springer, 2012.

[Kleinberg et al., 2017] Jon Kleinberg, Sendhil Mullainathan, and Manish Raghavan. Inherent trade-offs in the fair determination of risk scores. Innovations in Theoretical Computer Science, 2017

[Kleinberg et al., 2018] Jon Kleinberg, Jens Ludwig, Sendhil Mullainathan, and Cass R Sunstein. Discrimination in the age of algorithms. Journal of Legal Analysis, 10,2018

[Kull and Flach, 2014] Meelis Kull and Peter Flach. Patterns of dataset shift. In First International Workshop on Learning over Multiple Contexts (LMCE) at ECML PKDD, 2014.

[Moreno-Torres et al., 2012] Jose G Moreno-Torres, Troy Raeder, RocíO AlaizRodríGuez, Nitesh V Chawla, and Francisco Herrera. A unifying view on dataset shift in classification. Pattern Recognition, 45(1):521-530, 2012.

[Pleiss et al., 2017] Geoff Pleiss, Manish Raghavan, Felix Wu, Jon Kleinberg, and Kilian Q Weinberger. On fairness and calibration. In Advances in Neural Information Processing Systems, pages 5684-5693, 2017.

[ProPublica, 2016] ProPublica. COMPAS Recidivism Risk Score Data \& Analysis. github.com/propublica/compas-analysis, 2016.

[Romei and Ruggieri, 2014] Andrea Romei and Salvatore Ruggieri. A multidisciplinary survey on discrimination analysis. The Knowledge Engineering Review, 29(5):582-638, 2014.

[Saerens et al., 2002] Marco Saerens, Patrice Latinne, and Christine Decaestecker. Adjusting the outputs of a classifier to new a priori probabilities: a simple procedure. Neural computation, 14(1):21-41, 2002.

[Woodworth et al., 2017] Blake Woodworth, Suriya Gunasekar, Mesrob I Ohannessian, and Nathan Srebro. Learning non-discriminatory predictors. In Conference on Learning Theory, pages 1920-1953, 2017.

[Zafar et al., 2017a] Muhammad Bilal Zafar, Isabel Valera, Manuel Gomez Rodriguez, and Krishna P Gummadi. Fairness beyond disparate treatment \& disparate impact: Learning classification without disparate mistreatment. In International Conference on World Wide Web, pages 1171-1180, 2017.

[Zafar et al., 2017b] Muhammad Bilal Zafar, Isabel Valera, Manuel Gomez Rodriguez, and Krishna P Gummadi. Fairness constraints: Mechanisms for fair classification. In Artificial Intelligence and Statistics, pages 962-970, 2017. 
[Zemel et al., 2013] Rich Zemel, Yu Wu, Kevin Swersky, Toni Pitassi, and Cynthia Dwork. Learning fair representations. In Proceedings of the 30th International Conference on Machine Learning (ICML-13), pages 325-333, 2013.

[Zhang et al., 2018a] Brian Hu Zhang, Blake Lemoine, and Margaret Mitchell. Mitigating unwanted biases with adversarial learning. In Proceedings of the AAAI/ACM Conference on AI, Ethics, and Society, AIES, pages 335-340. ACM, 2018.
[Zhang et al., 2018b] Lu Zhang, Yongkai Wu, and Xintao Wu. Achieving nondiscrimination in prediction. In International Joint Conference on Artificial Intelligence, IJCAI, pages 3097-3103, 2018.

[Zliobaite and Custers, 2016] Indre Zliobaite and Bart Custers. Using sensitive personal data may be necessary for avoiding discrimination in data-driven decision models. Artif. Intell. Law, 24(2):183-201, 2016. 